\title{
Charge Carrier Transport Properties in Single-Walled Carbon Nanotube Fibers
}

\author{
V.K. Ksenevich ${ }^{a, *}$, D. Seliuta ${ }^{b}$, Z. Martūnas ${ }^{b}$, \\ I. KašAlynas ${ }^{b}$, G. VAlušis ${ }^{b}$, J. GaliberT $^{c}$, M.E. Kozlov $^{d}$ \\ AND V.A. SAMUILOV \\ ${ }^{a}$ Department of Physics, Belarus State University \\ Nezalezhnasti av. 4, 220030 Minsk, Belarus \\ ${ }^{b}$ Semiconductor Physics Institute \\ A. Goštauto 11, LT-01108 Vilnius, Lithuania \\ ${ }^{c}$ Laboratoire de Physique de la Matière Condensée, S.N.C.M.P.-I.N.S.A. \\ 135, Av. de Rangueil, 31077 Toulouse CEDEX 4, France \\ ${ }^{d}$ The Nano Tech Institute, University of Texas at Dallas \\ P.O. Box 830688, BE26, Richardson, TX 75083-0688, USA
}

${ }^{e}$ Department of Materials Science, State University of New York at Stony Brook

$$
\text { N.Y. 11794-2275, USA }
$$

\begin{abstract}
Transport properties via temperature dependences of sample resistance $R(T)$ and influence of microwave field of $10 \mathrm{GHz}$ on the conductivity of the single-walled carbon nanotubes fibers are investigated. The $R(T)$ dependences studied within $4.2-300 \mathrm{~K}$ can be well approximated by the Mott law for $3 \mathrm{D}$ variable range hopping below $T=80 \mathrm{~K}$ and by typical law for fluctuation-induced tunnelling model within the temperature range 80 $300 \mathrm{~K}$. We associate the observed increase in the conductivity with microwave power by increase in hopping probability of the charge carriers between single-walled carbon nanotubes.
\end{abstract}

PACS numbers: 73.63.Fg

\section{Introduction}

Transport properties of single-walled carbon nanotubes (SWCNTs) are of particular interest from a point of view of both basic physics and applications. On the one hand, the material allows a study of a large variety of different quan-

*corresponding author; e-mail: ksenevich@bsu.by 
tum phenomena like single-electron tunnelling [1], Luttinger liquid behaviour [2], ballistic transport [3], etc. On the other hand, carbon nanotube-based electronics has promising application starting from antistatic shielding, transparent conductive layers, interconnections and heat sinks in integrated circuits as well as gas, bio- and chemical sensors. For the latter aims a particular role is played by arrays (fibers) of carbon nanotubes. Hence, the understanding of charge carrier properties is one of the key-topics in the development of new devices.

\section{Experimental results}

The SWCNTs fibers were synthesized using wet spinning process from solutions comprising nanotubes, surfactant and water. This process provides polymerfree nanotubes fibers without the need for super acid. The fabrication procedure was described in detail in Ref. [4]. The SWCNT raw material itself was prepared by laser ablation. The fibers consist of a great number of individual nanotubes and typically have a diameter of 50-100 $\mu \mathrm{m}$. Scanning electron microscopy investigations of the structure of the fibers surface suggest a certain degree of individual nanotubes alignment inside the fibers.

In order to determine transport mechanisms, the temperature dependences of resistance $R(T)$ of SWCNT fibers were measured in the range of 4.2-300 K. To complete charge carrier transport picture in strong electric fields, we have applied microwave (MW) field of $10 \mathrm{GHz}$ to measure change of the conductivity averaged over the MW field period. The dependences were recorded at temperatures $77 \mathrm{~K}$ and $300 \mathrm{~K}$.

\section{Discussion}

The $R(T)$ dependence of the SWCNT fibers is shown in Fig. 1. In the lowtemperature range $(4.2-80 \mathrm{~K})$ the resistance as a function of temperature exhibits a classical 3D variable range hopping Mott conduction, following the law [5]:

$$
R=R_{0} \exp \left(T_{0} / T\right)^{1 / 4} .
$$

In the temperature range $T=80-300 \mathrm{~K} R(T)$ dependences can be approximated by a typical law for fluctuation-induced tunnelling conductivity mechanism $[6,7]$ :

$$
R=R_{0} \exp \left(\frac{T_{1}}{T+T_{0}}\right),
$$

where parameters $T_{0}$ and $T_{1}$ are defined by the following equations:

$$
\begin{aligned}
& T_{0}=16 \varepsilon_{0} \hbar A V_{0}^{3 / 2} /\left[\pi e^{2} k_{\mathrm{B}}\left(2 m_{\mathrm{e}}\right)^{1 / 2} w^{2}\right], \\
& T_{1}=8 \varepsilon_{0} A V_{0}^{2} /\left(e^{2} k_{\mathrm{B}} w\right),
\end{aligned}
$$

where $w$ is the width of the tunnel junction, $A$ labels its area, and $V_{0}$ is the height of the contact potential; $k_{\mathrm{B}}$ stands for the Boltzmann constant, $e$ is the electronic charge and $m_{\mathrm{e}}$ denotes the electron's effective mass, and $\varepsilon_{0}$ the vacuum permittivity. 


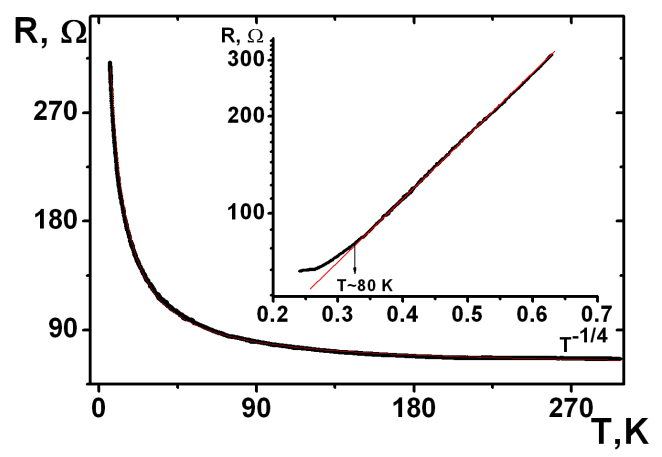

Fig. 1. The temperature dependence of the resistance of the SWCNT fibers. The inset shows the $\log R-T^{-1 / 4}$ plot.

Differently from systems with hopping charge carrier transport between localized sites, the fluctuation-induced tunnelling conductivity model was proposed for disordered heterogeneous systems. As it is known [6], the conduction electrons are delocalized here and free to move over distances very large as compared to atomic dimensions. In these systems (for example, conductor-insulator composites, granular metals, disordered semiconductors) electron transfer between large conductive segments separated by small insulating gaps dominates electrical conductivity. In our case fitting results agree well with experiment data indicating thus importance of intertube contacts between individual carbon nanotubes. We assumed that the area $A$ of the junction equals the cross section of a SWCNT with diameter of about $1.5 \mathrm{~nm}$. Using the expressions (3) and (4) the effective junction width is estimated to be $4.6 \mathrm{~nm}$ and the effective barrier height to be $0.172 \mathrm{eV}$.

The conductivity of SWCNT fibers increased when the microwave field of $10 \mathrm{GHz}$ was applied to the samples. The dependences of the variation of the conductivity of SWCNT fibers on applied MW field power at the temperatures 77 and $300 \mathrm{~K}$ are shown in Fig. 2.

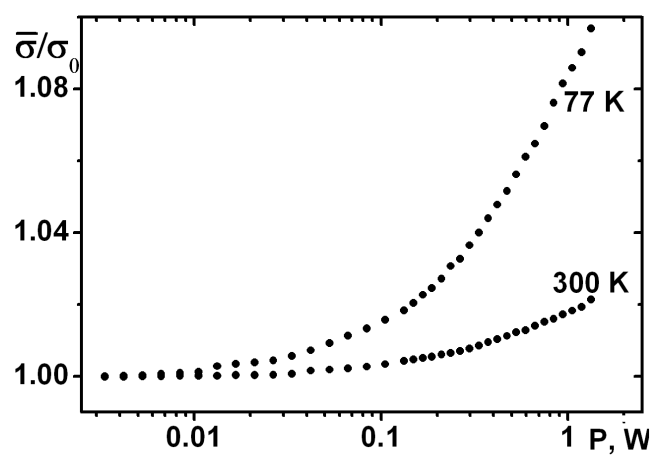

Fig. 2. The dependence of the conductivity variation on applied MW power. 
The $R(T)$ dependences indicate that the conduction of the SWCNT fibers can be considered as a combination of two mechanisms: variable range hopping [5] which prevails in the low-temperature range and fluctuation-induced tunnelling due to existence of the contact barriers between individual nanotubes inside the fibers [7]. The energy barriers can exist as a result of contacts either between metallic and semiconductive nanotubes or between semiconductive nanotubes with different band gap.

The increase in the conductivity on MW power becomes more essential with the temperature decrease as one can see from Fig. 2. At the same MW power of about $1 \mathrm{~W}$ the relative conductivity increase was about $2 \%$ and $10 \%$ at $300 \mathrm{~K}$ and $77 \mathrm{~K}$, correspondingly. According to $\mathrm{R}(\mathrm{T})$ dependences the VRH starts to prevail at temperatures below $80 \mathrm{~K}$. Therefore we associate the increase in the conductivity by increase in the hopping probability of charge carriers in MW field.

In conclusion, charge carrier properties in SWCNT fibers were studied. Experimental data are consistent both with the charge transport model for homogeneously disordered materials due to low-temperature localization of charge carriers arising from the defects in the tubes themselves and with the model for heterogeneous materials that emphasises the role of energy barriers between the nanotubes.

\section{Acknowledgments}

The work was supported by the Foundation of Basic Research of Republic of Belarus (grant No. F07F-013), NATO Collaborative Linkage Grant (reference CBP.EAP.CLG982007) and Lithuanian State Science and Studies Foundation under contract V-07018.

\section{References}

[1] M. Bockrath, D.H. Cobden, P.L. McEuen, N.G. Chopra, A. Zettl, A. Thess, R.E. Smalley, Science 275, 1922 (1997).

[2] M. Bockrath, D.H. Cobden, J. Lu, A.G. Rinzler, R.E. Smalley, L. Balents, P.L. McEuen, Nature 397, 598 (1999).

[3] A. Javey, J. Lu, A.G. Rinzler, R.E. Smalley, L. Balents, P.L. McEuen, Nature 424, 654 (2003).

[4] M.E. Kozlov, R.C. Capps, W.M. Sampson, V.H. Ebron, J.P. Ferraris, R.H. Baughman, Adv. Materials 17, 614 (2005).

[5] B.I. Shklovskii, A.L. Efros, Electronic Properties of Doped Semiconductors, Springer-Verlag, Berlin 1984.

[6] P. Sheng, Phys. Rev. B 21, 2180 (1980).

[7] G.T. Kim, S.H. Jhang, J.G. Park, S. Roth, Synth. Metals 117, 123 (2001). 